\title{
Hypomagnesemia in diabetes patients: comparison of serum and intracellular measurement of responses to magnesium supplementation and its role in inflammation
}

This article was published in the following Dove Press journal: Diabetes, Metabolic Syndrome and Obesity:Targets and Therapy

\author{
Nadia Zghoul' \\ Nada Alam-Eldin' \\ Ivan Tong Mak $^{2}$ \\ Burton Silver 3 \\ William B Weglicki² \\ 'Clinical Research Unit, Dasman \\ Diabetes Institute, Dasman, Kuwait, \\ Kuwait; ${ }^{2}$ Department of Biochemistry \\ and Molecular Medicine, George \\ Washington University, Washington, \\ DC, USA; Intracellular Diagnostics, \\ Inc. ${ }^{\circledR}$, Medford, OR, USA
}

\begin{abstract}
Purpose: In this clinical trial, we assessed the efficacy of magnesium $(\mathrm{Mg})$ supplementation in hypomagnesemic type 2 diabetes patients in restoring serum and intracellular $\mathrm{Mg}$ levels. The study had two coprimary end points: the change in serum and intracellular $\mathrm{Mg}$ level between baseline and after 3 months of supplementation. We compared the efficacy with regard to lowering hemoglobin A1c (HbA1c), C-reactive protein (CRP), tumor necrosis factor- $\alpha$ (TNF- $\alpha$ ), and 8-isoprostane as secondary end points.
\end{abstract}

Patients and methods: In an open-label trial, 47 hypomagnesemic type 2 diabetes patients were administered $336 \mathrm{mg} \mathrm{Mg}$ daily. At baseline and after 3 months, serum, cellular Mg, and inflammation biomarkers were measured. For intracellular $\mathrm{Mg}$ levels, sublingual epithelial cells were analyzed by analytical scanning electron microscopy using computerized elemental $\mathrm{X}$-ray analysis. Blood samples were analyzed for $\mathrm{Mg}$, creatinine, HbA1c, and CRP. Systemic inflammatory markers including TNF- $\alpha$ and the oxidative stress marker 8-isoprostane were determined using enzyme-linked immunosorbent assay.

Results: Mg supplementation significantly increased the intracellular and serum levels. Statistically clinical improvement in HbA1c and CRP levels was not observed, but significant decreases in TNF- $\alpha$ as well as in 8-isoprostane were found.

Conclusion: A feasible clinical method for the assessment of intracellular $\mathrm{Mg}$ was demonstrated in tissue samples obtained noninvasively, providing evidence for potential clinical translation of this method to routinely determine intracellular $\mathrm{Mg}$ concentration.

Keywords: hypomagnesemia, type 2 diabetes, intracellular magnesium, elemental X-ray analysis

\section{Introduction}

Hypomagnesemia contributes to the pathophysiology of diabetes and metabolic syndrome. ${ }^{1,2}$ In one study, there was a graded inverse relationship between serum magnesium $(\mathrm{Mg})$ levels and incident type 2 diabetes; this association remained significant even after adjusting for potential confounders, including diuretic use. ${ }^{3}$ This relationship implies a clear need to consider $\mathrm{Mg}$ as a potential supplement for treating type 2 diabetes. Further, there is abundant evidence demonstrating the prevalence and adverse clinical consequences of $\mathrm{Mg}$ deficiency in patients with diabetes mellitus. It would be prudent for physicians treating such patients to consider Mg deficiency as a contributing factor in many diabetic complications. ${ }^{4}$

The lower normal serum Mg level at the clinical laboratory in Dasman Diabetes Institute (DDI; Dasman, Kuwait) is $0.74 \mathrm{mmol} / \mathrm{L}$, but this level only represents $<1 \%$ of
Correspondence: Nadia Zghoul

Clinical Research Unit, Dasman Diabetes

Institute, PO Box II 80 Dasman, 15462

Kuwait, Kuwait

Tel +96522242999

Fax +96522492406

Email nadia.zghoul@dasmaninstitute.org 
the total body $\mathrm{Mg}$ level. Importantly, a significant proportion of patients with normal serum Mg levels may have cellular $\mathrm{Mg}$ deficiency because the highest level of $\mathrm{Mg}$ is in skeletal muscles, bones, and other organs. Thus, intracellular Mg levels compared with serum $\mathrm{Mg}$ levels are more representative end points of total body $\mathrm{Mg}$ deficiency. Sublingual epithelial cells are a rapidly renewing, homogeneous cell population that reflects the current total body intracellular mineral status. A study of intracellular $\mathrm{Mg}$ levels demonstrated that $\mathrm{Mg}$ levels in sublingual epithelial cells correlated better with $\mathrm{Mg}$ levels in heart tissue (obtained during bypass surgery) than with serum Mg levels., ${ }^{5,6}$

Our results may support a wider application of the cellular Mg assay not only in patients with diabetes or metabolic syndrome but also in those with cancer, cardiovascular disease, or other diseases where $\mathrm{Mg}$ and glucose homeostasis may be impaired.

The anti-inflammatory effects of oral Mg supplementation in patients with diabetes or metabolic syndrome and those with low serum Mg levels have been studied with increased interest in recent years. ${ }^{1,7}$ In the present open-label clinical trial, 47 hypomagnesemic patients with type 2 diabetes were enrolled; for 3 months, they were administered Mg-l-lactate tablets that provided $336 \mathrm{mg}$ of $\mathrm{Mg}$ daily. The repletion of $\mathrm{Mg}$ levels in serum and oral epithelial cells was compared with changes in C-reactive protein (CRP), tumor necrosis factor- $\alpha$ (TNF- $\alpha$ ), hemoglobin A1c (HbA1c), and the oxidative stress biomarker 8-isoprostane.

\section{Subjects, materials, and methods Patients and eligibility criteria}

At the DDI, from March 2015 until April 2016, the records of 1022 patients with type 2 diabetes were screened for inclusion. Inclusion criteria required the presence of hypomagnesemia (serum levels $<0.74 \mathrm{mmol} / \mathrm{L}$ ), aged between 21 and 70 years, and the presence of at least one abnormal indicator of inflammation. Patients were excluded if they suffered from significant gastrointestinal disorders (eg, chronic diarrhea), chronic inflammatory disorders (eg, psoriasis), impaired renal function (estimated glomerular filtration rate [eGFR] $<60$ $\mathrm{mL} / \mathrm{min}$ ), hypertension (eg, angiotensin-converting enzyme inhibitors and diuretics), heart block (eg, electrocardiographic [ECG] evidence of left bundle branch block), or heart failure (eg, treated with aldosterone antagonists). Patients who had used $\mathrm{Mg}$ supplements within 3 months and pregnant women were also excluded.

This study was conducted in accordance with the Good Clinical Practice criteria and according to the tenets of the
Declaration of Helsinki of 2013. The study protocol and informed consent form were approved by the ethics committee of the DDI before initiating the study. All patients provided written informed consent before inclusion, in compliance with 21 case report forms (CRF) Part 50, and their data were recorded in CRF specific for the study including an adverse event questionnaire.

Further, patients were classified according to the World Health Organization obesity classification guidelines (obese when the body mass index [BMI] was $\left.\geq 30.0 \mathrm{~kg} / \mathrm{m}^{2}\right) .{ }^{8}$

\section{Study design}

This was an open-label clinical trial in which Mg-l-lactate was supplemented for 12 weeks in 47 patients with type 2 diabetes and documented hypomagnesemia. Patients initially underwent a clinical and laboratory evaluation, and blood samples were obtained for $\mathrm{HbAlc}$ and creatinine level estimation and for assays of serum and cellular $\mathrm{Mg}$ level determination. After 12 weeks, the patients underwent a subsequent evaluation. The study was registered under https:// clinicaltrials.gov/ct2/show/NCT01980459.

The trial design was changed from an initially proposed randomized parallel group placebo study to an open-label trial because of the discoloration of placebo tablets; since the placebo tablets were discolored, patients would be aware that they were in the placebo arm. Therefore, the number of groups was reduced from two to one with 50 patients. Study end points, patient screening, the $\mathrm{Mg}$ tablet supplementation protocol, and laboratory assessments remained the same as in the original proposal. The change in design was approved by the ethical review committee at the DDI (Kuwait) and by the Office of Human Research-Institutional Review Board at George Washington University (Washington, DC, USA).

\section{Intervention}

Patients received $\mathrm{Mg}$ supplementation twice a day; they received $336 \mathrm{mg}$ of elemental $\mathrm{Mg}$, which approximates the recommended daily allowance for adults. $\mathrm{Mg}$ tablets $(7 \mathrm{mEq})$ were provided by Niche Pharmaceuticals, Inc. (Southlake, TX, USA; Lot \# B060057BA). This formulation of Mg-llactate has been reported to have a bioavailability of $41 \%$ (compared with $\mathrm{Mg}$ oxide), and its sustained release has a low risk of gastrointestinal side effects. The longer time (3 months) of treatment is based on a prior $\mathrm{Mg}$ treatment trial of hypomagnesemic patients who achieved a $20 \%$ increase in serum Mg levels after 3 months. ${ }^{9}$

At each clinic visit, patients were provided with sufficient tablets for 1 month of treatment. Data were recorded in the CRF 
at each clinic visit. Compliance was assessed by counting the number of tablets, and patients returning $<20 \%$ of the tablets were considered compliant. Patients were asked to continue their regular medication and diet as usual and were advised not to take any other supplements that might contain $\mathrm{Mg}$.

\section{Primary and secondary end points}

The study had two coprimary end points: the change in $\mathrm{Mg}$ levels in the serum and mucosal cells between the baseline and the 3-month visit. The change in the following secondary end points from the baseline was also measured at the 3-month visit: CRP, TNF- $\alpha, 8$-isoprostane, and HbA1c.

At the initiation of the clinical trial, assays of serum and cellular $\mathrm{Mg}$ and inflammatory biomarker levels were performed; serum $\mathrm{Mg}$ levels were monitored at 1 and 2 months. Three months later, both tests were repeated. Body weight, blood pressure, and heart rate were measured at every clinic visit. ECG was conducted at the screening visit and at the 2- and 3-month visits for safety. Any ECG abnormality was recorded in the CRF.

\section{Clinical laboratory blood tests}

Clinical laboratory tests were performed for each patient before the start of $\mathrm{Mg}$ supplementation (screening/baseline) and at the final patient visit. Assays of serum $\mathrm{Mg}, \mathrm{HbAlc}$, and CRP levels were performed. Serum $\mathrm{Mg}$ and $\mathrm{HbA} 1 \mathrm{c}$ levels were monitored at 1 and 2 months after the treatment was started, and all tests were repeated at the final visit after 3 months.

\section{Intracellular Mg analysis or epithelial cell mineral analysis}

Sublingual epithelial cells were noninvasively obtained from each patient and were fixed on slides before performing assays for $\mathrm{Mg}$ levels by analytical scanning electron microscopy (ASEM) using the computerized elemental $\mathrm{X}$-ray analysis programs. Patient cell samples before and after $\mathrm{Mg}$ treatment were obtained at the baseline and at the end of 3 months.

\section{Sublingual epithelial cell specimen collection}

The patients fasted overnight and were asked to rinse their mouth with water; sublingual soft tissue on the floor of the mouth was scraped using a wooden spatula. Cells were transferred on specially prepared slides (Intracellular Diagnostics, Inc., Medford, OR, USA). Immediately, the cells were fixed chemically (Pap-Pak ${ }^{\circledR}$ Cytology Fixative; Camarillo, CA,
USA), allowed to dry, and stored at $4^{\circ} \mathrm{C}$ until analysis. All collected samples were shipped to Dr. Burton Silver (Intracellular Diagnostics, Inc.), who was our collaborator.

Each epithelial cell sample was viewed using ASEM and was analyzed by the elemental energy-dispersive X-ray analysis method. This technique uses energy-dispersive X-ray microanalysis (EXAtm) for the noninvasive intracellular measurement of $\mathrm{Mg}$ and potassium (K) (USA patent Number: 4-717-826, B.S.) levels. Sublingual epithelial cells were chosen as markers as they are easily accessible, noncornified, and aerobic; they have a high cytoplasm-to-nucleus ratio, turnover in $<3$ days, long shelf life, and they exhibit 99\% viability. The excitation of cellular atoms displaces inner orbital electrons, which are replaced by electrons from higher energy cells releasing fluoresced X-rays, which allows the quantitation of intracellular elements. EXAtm units equal the X-ray intensity (peak divided by background) divided by unit cell volume. EXAtm units are converted to milliequivalents per liter by a conversion constant for each element derived from a reference standard of a highly stable matrix synthetic glass containing known amounts of the elements to be analyzed. The standard was certified by the National Bureau of Standards (Washington, DC, USA) and Corning Diagnostics (Cambridge, MA, USA).

\section{Oxidative stress and inflammatory biomarkers \\ 8-Isoprostane, TNF- $\alpha$, and CRP}

Plasma samples were collected from ethylenediaminetetraacetic acid-treated blood samples obtained at the baseline for all patients and at their final visit after 3 months of treatment with Mg. The levels of inflammatory biomarkers (CRP and TNF- $\alpha$ ) and the oxidative reactive oxygen species (ROS) stress biomarker 8-isoprostane were assessed.

All plasma samples were stored at $-80^{\circ} \mathrm{C}$ before analysis. The levels of the biomarkers were measured using sandwich enzyme-linked immunosorbent assay kits, and color intensity was read on a plate reader at the recommended wavelength following the instruction manual for each kit. A multimode microplate reader was used for measuring absorbance (Synergy H4; BioTek Instruments, Winooski, VT, USA), and the software Gen5 $5^{\mathrm{TM}} 1.10$ was used for analysis.

For 8-isoprostane detection, plasma samples were treated further with $0.005 \%$ butylated hydroxytoluene (SigmaAldrich Corp., Albuch, Germany) before being frozen. 8-Isoprotane levels were assessed using an 8-isoprostane Enzyme immunoassay (EIA) kit (Catalog No. 516351; Cayman Chemical, Ann Arbor, MI, USA). 
Absorbance readings were averaged, nonspecific binding was subtracted, and the four-parameter logistic (4-PL) regression curve fit was generated for standards with known concentration; the sample concentration was interpolated, and the dilution factor used was corrected according to assay kit instructions. The software GraphPad Prism was used for the 4-PL fit of the standard samples to then extrapolate the sample concentrations.

\section{Statistical analyses}

Changes in the mean levels of the primary and secondary end points were tested using paired $t$-tests. Most data were expressed as mean $\pm \mathrm{SD}:{ }^{*} p<0.05, * * p<0.01$, and $* * * p<0.001$.

\section{Results}

Of the 1022 patients with type 2 diabetes who were screened, 225 met the renal inclusion criteria (creatinine level $<140 \mu \mathrm{mol} / \mathrm{L}$, eGFR $>60 \mathrm{~mL} / \mathrm{min} / 1.73 \mathrm{~m}^{2}$ ): 77 refused to take screening for Mg levels, 61 had normal Mg levels, and 87 had hypomagnesemia (average serum $\mathrm{Mg}$ level $=0.68 \pm 0.06 \mathrm{mmol} / \mathrm{L}$ ) and CRP levels (average $=0.32 \pm 0.54$ ). Of the 62 patients who agreed to participate, 47 completed the trial, eight did not receive the allocated intervention and were excluded from the trial mainly due to abnormal ECG results, and seven dropped out of the study. The 47 study patients were classified according to their BMI, with 15 patients classified as nonobese and 32 as obese.

Ultimately, data from the 47 patients were analyzed, with intracellular Mg levels analyzed in 46 patients and isoprostane levels analyzed in 25 patients. The study flow diagram is illustrated in Figure S1.

\section{Demographics and clinical characteristics}

Table S1 summarizes the demographic and clinical characteristics of all patients studied (25 [53\%] male and 22 [47\%] female patients; 32 [68\%] obese and 15 [32\%] nonobese).
The average patient age was 52 years (range, 27-69 years) with the majority of the patients (22) within the 50-59 years of age range.

\section{Primary and secondary outcomes}

Table 1 shows the results of the clinical laboratory and biochemical parameters assessed for all the patients at baseline and at the end of the trial after completing their final visit. The laboratory values for all the patients are further listed dividing the patients in obese and nonobese groups. At the end of the 3-month supplementation period with an average of $22.8 \mathrm{~g}$ of Mg-1-lactate per patient, both obese and nonobese Mg-supplemented subjects showed significant increase in their serum $\mathrm{Mg}$ levels $(0.71 \pm 0.06$ $\mathrm{mmol} / \mathrm{L}$ to $0.73 \pm 0.06 \mathrm{mmol} / \mathrm{L}, p<0.0001)$ and also significant increase in their intracellular Mg levels $(33.1 \pm 1.25$ $\mathrm{mEq} / \mathrm{L}$ to $35.55 \pm 5.6 \mathrm{mEq} / \mathrm{L}, p<0.01)$. Patients also showed a significant reduction in their TNF- $\alpha$ levels from $9.1 \pm 1.7$ to $6.1 \pm 2.7(p<0.0001)$. Metabolic control as measured by HbA1c however was not altered significantly (HbA1c, $8.1 \pm 1.7 \%$ vs $7.9 \pm 1.9 \%$ ), and CRP also showed no significant changes at the end of the supplementation period.

Review of the serum $\mathrm{Mg}$ mean values pre- and posttreatment shows a significant difference in both groups, but only reaching the normal level of $0.74 \mathrm{mEq} / \mathrm{L}$ in the nonobese group (Figure $1 \mathrm{~A}$ and $\mathrm{B}$ ); the intracellular $\mathrm{Mg}$ mean values showed that both obese and nonobese patients had significant increases above the normal level of $34 \mathrm{mEq} / \mathrm{L}$ (Figure $1 \mathrm{C}$ and D). When comparing serum and intracellular $\mathrm{Mg}$ levels before and after treatment and correlating it to patient age, no statistical significance was noted among the different age groups neither in the basal levels of the patients nor in their response to $\mathrm{Mg}$ supplementation.

For the inflammatory biomarkers, both TNF- $\alpha$ and CRP data were analyzed. TNF- $\alpha$ decreased (Figure $2 \mathrm{~A}$ ), while no

Table I Laboratory evaluation before and after treatment of the study population at baseline and at the end of 3-month therapy

\begin{tabular}{|c|c|c|c|c|c|c|}
\hline \multirow[t]{2}{*}{ Outcome } & \multicolumn{2}{|c|}{ Patients $(n=47)$} & \multicolumn{2}{|c|}{ Obese group $(n=32)$} & \multicolumn{2}{|c|}{ Nonobese group $(n=15)$} \\
\hline & Baseline & End & Baseline & End & Baseline & End \\
\hline $\mathrm{HbAlc}(\%)(\mathrm{mmol} / \mathrm{mmol})$ & $\begin{array}{l}8.1 \pm 1.7 \\
(64.6 \pm 18.8)\end{array}$ & $\begin{array}{l}7.9 \pm 1.6 \\
(63.4 \pm 17.0)\end{array}$ & $\begin{array}{l}8.4 \pm 1.8 \\
(67.8 \pm 19.6)\end{array}$ & $\begin{array}{l}8.1 \pm 1.6 \\
(64.8 \pm 17.9)\end{array}$ & $\begin{array}{l}7.5 \pm 1.5 \\
(57.7 \pm 15.5)\end{array}$ & $\begin{array}{l}7.6 \pm 1.4 \\
(60.2 \pm 15.1)\end{array}$ \\
\hline Magnesium (serum; mmol/L) & $0.7 I \pm 0.06$ & $0.73 \pm 0.06 * * * *$ & $0.70 \pm 0.06$ & $0.73 \pm 0.06 * * *$ & $0.7 I \pm 0.05$ & $0.74 \pm 0.05 * *$ \\
\hline Magnesium (intracellular; mEq/L) & $33.1 \pm 1.25$ & $35.5 \pm 5.6 * *$ & $32.9 \pm 1.2$ & $34.9 \pm 6.5 * * * *$ & $33.5 \pm 1.2$ & $36.8 \pm 2.1 * * *$ \\
\hline Potassium (intracellular; mEq/L) & $144.3 \pm 47.6$ & $184.7 \pm 73.4^{* *}$ & $144.7 \pm 48.7$ & $199.2 \pm 80.8 * *$ & $143.4 \pm 46.8$ & $154.7 \pm 43.1$ \\
\hline $\mathrm{CRP}(\mathrm{mg} / \mathrm{dL})$ & $0.59 \pm 0.65$ & $0.54 \pm 0.52$ & $0.58 \pm 0.58$ & $0.7 \pm 0.86$ & $0.61 \pm 0.8$ & $0.43 \pm 0.4$ \\
\hline TNF- $\alpha(p g / m L)$ & $9.1 \pm 1.7$ & $6.1 \pm 2.7^{* * * * *}$ & $9.1 \pm 1.9$ & $5.7 \pm 2.5^{* * * * *}$ & $9.1 \pm 1.25$ & $6.8 \pm 3.1 * *$ \\
\hline Isoprostane $(\mathrm{pg} / \mathrm{mL})$ & $91.7 \pm 21.8^{\#}$ & $71.7 \pm 27.5^{*}$ & $71.75 \pm 29.6$ & $58 \pm 27.8^{*}$ & $78.5 \pm 29.4$ & $60.1 \pm 30.5$ \\
\hline
\end{tabular}

Notes: Data are mean \pm SD. Values are mean \pm SD. ${ }^{*} \mathrm{n}=15$ for patients with isoprostane levels $>80 \mathrm{pg} / \mathrm{mL}$ at baseline. Serum magnesium normal reference range: $0.74-0.99$ $\mathrm{mmol} / \mathrm{L}$. Intracellular magnesium reference range: $34.0-42.0 \mathrm{mEq} / \mathrm{L}$. Potassium reference range: $80.0-240.0 \mathrm{mEq} / \mathrm{L}$. HbAlc reference range: $4 \%-6 \% \mathrm{NGSP}, 20-42 \mathrm{mmol} / \mathrm{mol}$ IFCC). $* p \leq 0.05$, $p$-value between baseline and end; $* * \leq 0.01 ; * * * \leq 0.001 ; * * * p \leq 0.000$ I.

Abbreviations: CRP, C-reactive protein; IFCC, International Federation of Clinical Chemistry; HbAIc, hemoglobin Alc; TNF- $\alpha$, tumor necrosis factor- $\alpha$. 

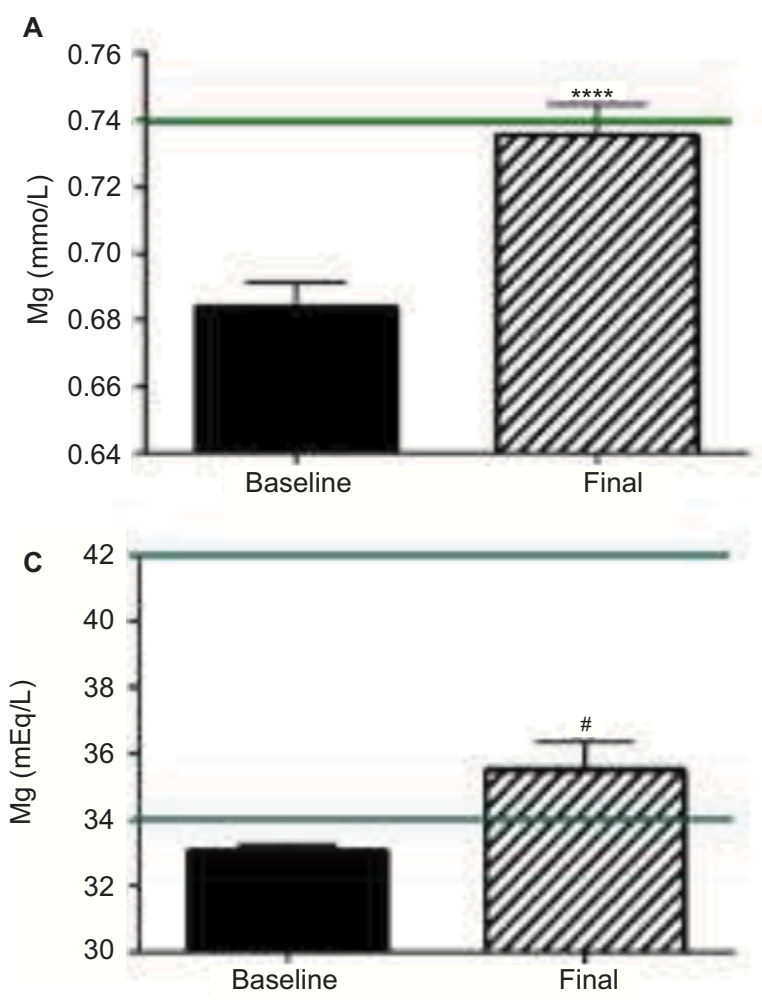

B

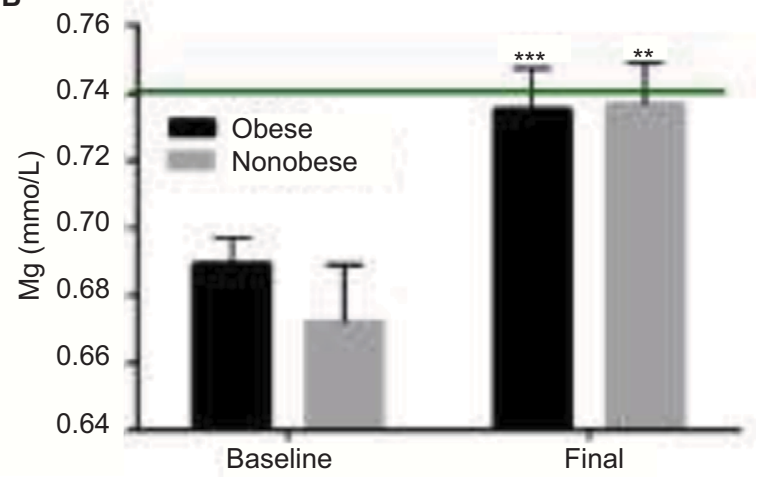

D

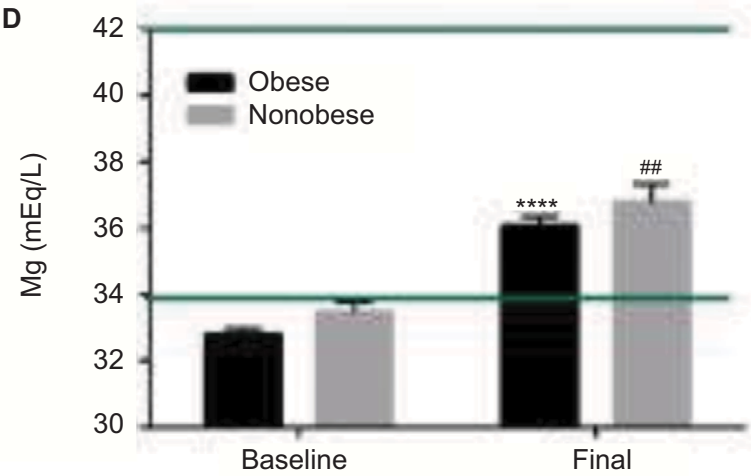

Figure I Serum magnesium levels (mmol/L) in (A) all patients after 12-week supplementation and (B) all patients grouped into nonobese or obese comparing two groups of all patients; intracellular magnesium levels $(\mathrm{mEq} / \mathrm{L})$ in $(\mathbf{C})$ all patients after 12 -week supplementation and (D) all patients grouped into nonobese or obese comparing two groups of all patients. ${ }^{* *} p=0.0019,{ }^{* * *} p=0.0003,{ }^{* * * *} p<000.1,{ }^{*} p=0.0077,{ }^{\#} p=0.0002$.

significant effect was observed in the CRP levels (Figure 2B). The decrease in TNF- $\alpha$ levels was evident in both subgroups (obese and nonobese); however, the decrease in the obese group was larger than in the nonobese group (Figure 2D). No significant effect was observed in the CRP levels of the subgroups (Figure 2E).

A significant decrease in isoprostane was observed in the patients with high baseline levels (Figure 2C); when subgrouping patients into obese and nonobese, the decrease in the obese group was statistically significant, but the decrease in the nonobese patients did not reach statistical significance (Figure $2 \mathrm{~F}$ ). The red line indicates the normal plasma 8-isoprostane level $(50 \mathrm{pg} / \mathrm{mL})$ normally seen in healthy individuals.

The elemental X-ray analysis procedure for the intracellular $\mathrm{Mg}$ determination provided quantitative data for the cellular mineral balance of several elements including $\mathrm{K}$, calcium, sodium, chloride, and phosphorus. Figure 3 shows the intracellular $\mathrm{K}$ levels at baseline and after 3 months of $\mathrm{Mg}$ supplementation in all patients (Figure 3A) and in patients subgrouped into obese and nonobese (Figure $3 \mathrm{~B}$ ). The reference range for intracellular $\mathrm{K}$ is $80.0-240 \mathrm{mEq} / \mathrm{L}$ ).

\section{Mg treatment compliance}

Overall, patient compliance was assessed by dividing the number of $\mathrm{Mg}$ tablets consumed (calculated by subtracting the number of tablets returned from the total number of tablets dispensed) by the total number of tablets prescribed. Four patients took $<80 \%$ of the dispensed Mg tablets (one of them took $<60 \%$ ).

\section{Adverse events}

All adverse events that occurred between the screening and last visit were recorded on the adverse event page of the CRF. Patients were able to comply with the twice-a-day dose schedule without significant side effects, and no dropouts were reported due to adverse events. Four patients reported mild-to-moderate gastrointestinal/abdominal symptoms, with a potential relationship to the intake of $\mathrm{Mg}$ tablets.

\section{Discussion}

Despite the evident role of hypomagnesemia in the outcome of diabetic morbidity, less clinical emphasis has been placed on the long-term treatment of hypomagnesemia in patients with diabetes. ${ }^{10}$ The link between $\mathrm{Mg}$ levels and diabetes 
A

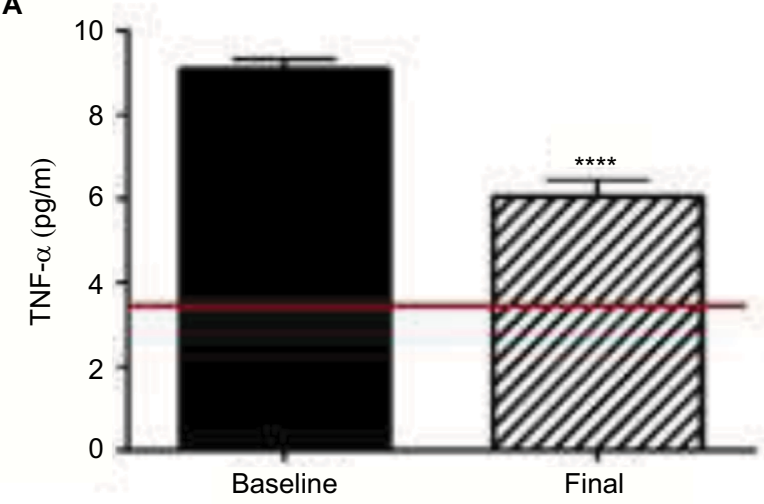

C
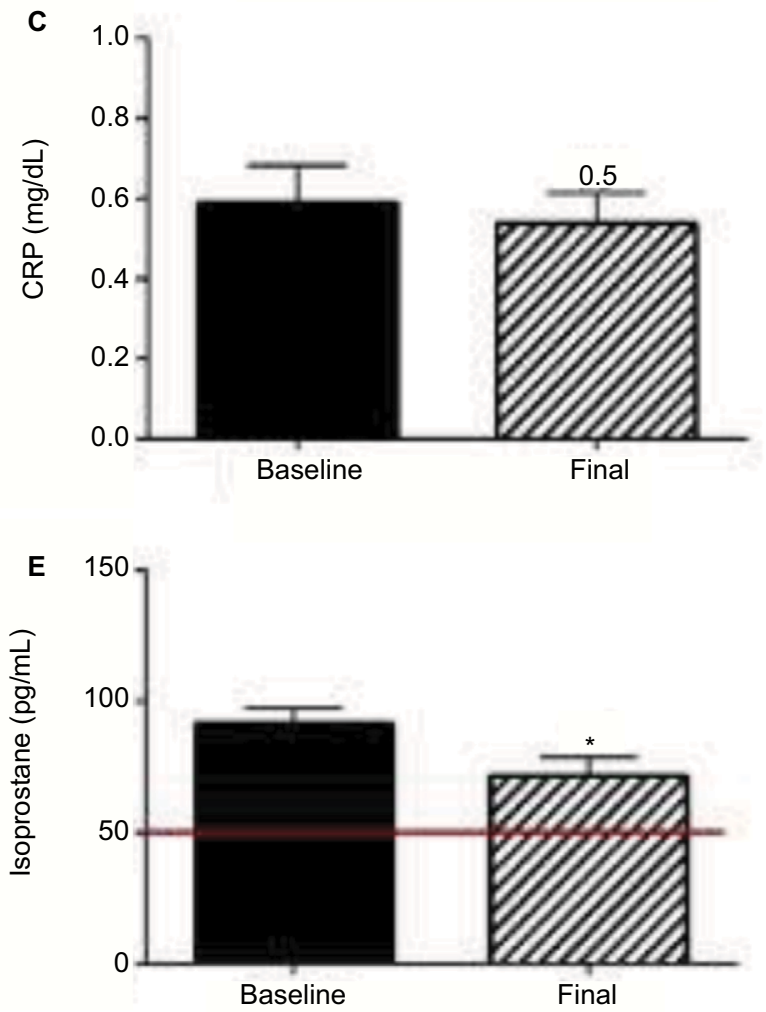

B

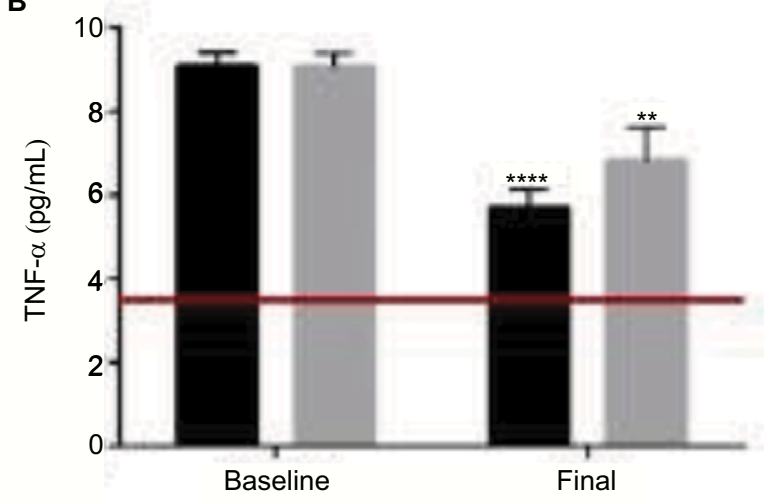

D
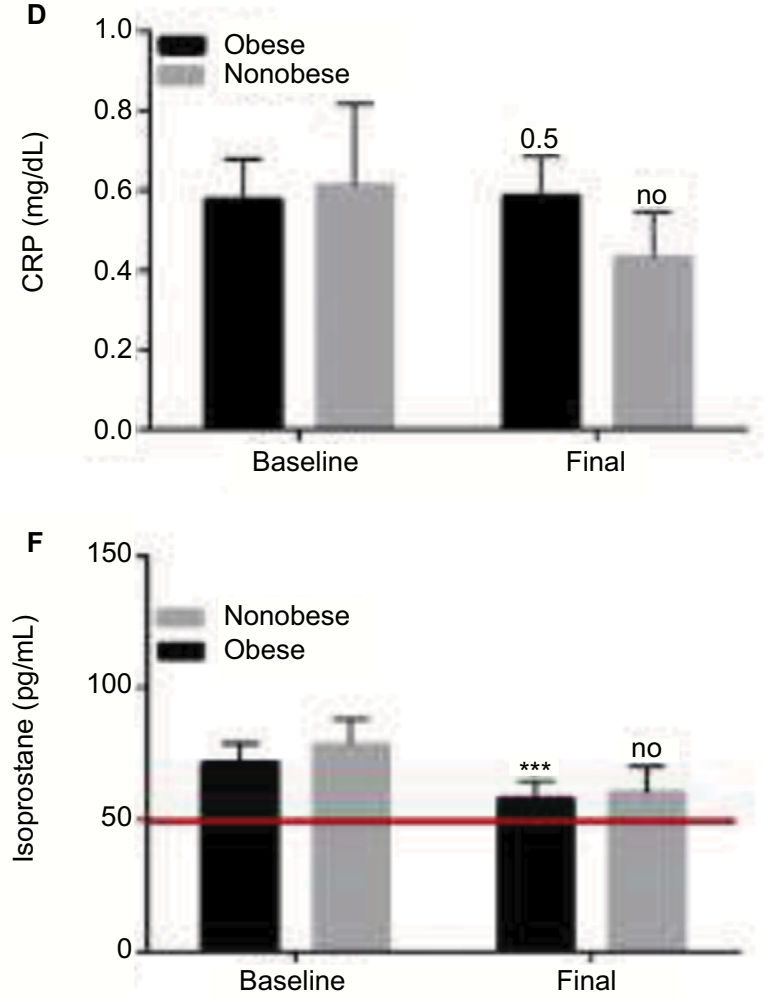

Figure 2 Serum levels of TNF- $\alpha$ (A), CRP (B), and isoprostane (C) after 3-month oral Mg supplementation in all patients and grouped into obese (black) and nonobese (gray) subjects (B, D, F). TNF- $\alpha$; **** $p<0.000$ I, ** $p=0.0060$. CRP was observed to be nonsignificant (ns). The red line indicates TNF- $\alpha$ level $\geq 3.5 \mathrm{pg} / \mathrm{mL}$ defined as low-grade inflammation. Isoprostane: ${ }^{*} p=0.0323 ; * * * p=0.0394$.

Abbreviations: CRP, C-reactive protein; TNF- $\alpha$, tumor necrosis factor- $\alpha$.

was strengthened in reports which demonstrated that oral Mg supplementation restored serum Mg levels, significantly improved plasma glucose levels after a 2-hour oral glucose tolerance test, and improved parameters of insulin sensitivity and metabolic control in patients with type 2 diabetes and with decreased serum Mg levels. ${ }^{11,12}$ Hypomagnesemia has been reported to potentially increase insulin resistance through a mechanism that is not yet clear; insulin resistance has also been associated with hypertension and atherosclerotic cardiovascular disease in several reports. ${ }^{13-15}$
A meta-analysis of randomized double-blind controlled trials by Song et al showed that oral $\mathrm{Mg}$ supplementation for 4-16 weeks may be effective in reducing plasma fasting glucose levels and raising high-density lipoprotein cholesterol in patients with type 2 diabetes, although the benefits on the long-term glycemic control as reflected by HbA1c levels remain to be determined. ${ }^{16}$

In an elegant review by Gommers et al, intracellular Mg was reported to play a role in the development of insulin resistance, with $\mathrm{Mg}$ affecting insulin receptor phosphorylation 
A

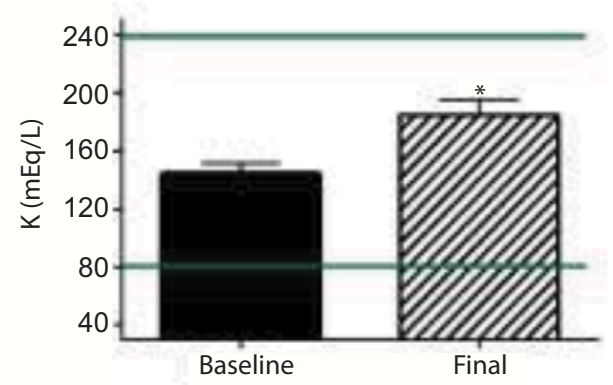

B

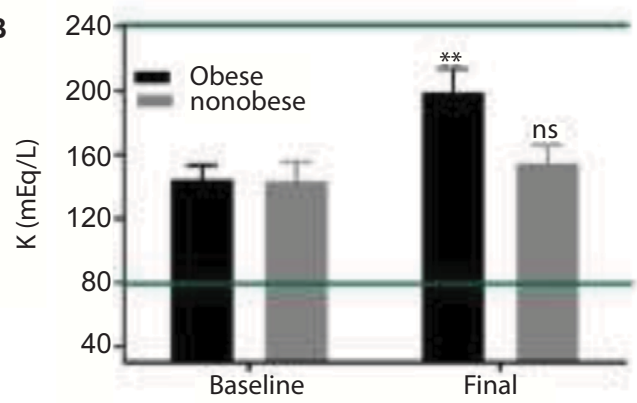

Figure 3 Intracellular potassium levels ( $\mathrm{mEq} / \mathrm{L}$ ) in all patients $(\mathbf{A})$ after 12 -week supplementation and all patients subgrouped into nonobese (gray) or obese (black) $* p=0.0030$ (B) comparing the two patient groups, ${ }^{* *} p=00025$.

Abbreviation: ns, nonsignificant.

and in turn hypomagnesemia enhancing insulin resistance. ${ }^{15}$ Nadler et al documented serum and erythrocyte intracellular $\mathrm{Mg}$ deficiency in patients with type 2 diabetes compared with healthy control subjects and showed that, in patients with type 2 diabetes, who received $400 \mathrm{mg}$ oral $\mathrm{Mg}$ supplementation daily for 8 weeks, intracellular $\mathrm{Mg}$ levels were restored to normal levels with no significant increase in serum Mg levels. The intracellular Mg levels were determined in erythrocytes by a nuclear magnetic resonance-based research technique. ${ }^{13}$

There is a clear need for an intracellular $\mathrm{Mg}$ assessment method that can be clinically applied. ${ }^{17}$ In our study, we assessed the efficacy of oral $\mathrm{Mg}$ supplementation on restoring cellular and serum $\mathrm{Mg}$ levels and the effects on circulating levels of CRP, TNF- $\alpha, 8$-isoprostane, and other glucose intolerance biomarkers in patients with type 2 diabetes.

The initial lower epithelial $\mathrm{K}$ levels observed in patients with type 2 diabetes might reflect the decreases in intracellular Mg levels. ${ }^{18} \mathrm{~A}$ decrease in intracellular $\mathrm{Mg}$, caused by $\mathrm{Mg}$ deficiency, was reported to release the Mg-mediated inhibition of the renal outer medullary potassium (ROMK) channels and increase $\mathrm{K}$ secretion, whereas normally intracellular Mg is suggested to bind to ROMK and block K efflux/ secretion. ${ }^{18}$ Our study revealed that $\mathrm{Mg}$ supplementation significantly elevated the intracellular K levels (Figure 3) might include these dual effects of the $\mathrm{Mg}$ stimulatory effect on the Na-K-ATPase and the blocking effect of $\mathrm{Mg}$ on the $\mathrm{K}$-efflux due to the ROMK channel. Mg deficiency may further impair Na-K-ATPase activity, which would contribute to $\mathrm{K}$ wasting and decreased cellular uptake of $\mathrm{K} .{ }^{19}$ The observed difference between the obese and nonobese groups could potentially imply that the Na-K-ATPase activity might be impaired more in the obese group, leading to the higher intracellular K levels.
The ability of Mg supplementation to diminish the levels of CRP, TNF- $\alpha$, and other biomarkers of inflammation and ROS/RNS stress has been previously reported..$^{20}$ However, Guerrero-Romero et al reported that only metabolic syndrome patients with severe hypomagnesemia $(<0.49 \mathrm{mmol} / \mathrm{L})$ had elevated levels of CRP and TNF- $\alpha .{ }^{21}$ In our study patients, the severity of hypomagnesemia was mild $(>0.49$ and $<0.74 \mathrm{mmol} / \mathrm{L}$ ), but we observed a significant decrease in TNF- $\alpha$ levels, while no significant effect was observed for CRP levels at the end of treatment.

Among our study patients, $85 \%$ were on statin therapy. It has been reported that statin therapy can affect plasma levels of inflammatory markers; statin therapy has been shown to specifically lower plasma levels of CRP levels. ${ }^{22-25}$ It is also known that statins have antioxidant effects by inhibiting the activity of oxidant enzymes, such as those of nicotinamide adenine dinucleotide phosphate oxidase and myeloperoxidase, and by upregulating the activity of antioxidant enzymes, eg, catalase, and enhancing endothelial nitric oxide bioavailability. ${ }^{26,27}$

Diabetes mellitus may also be associated with enhanced lipid peroxidation and persistent platelet activation. It has been shown that the in vivo formation of 8-isoprostane, which is a bioactive product of arachidonic acid peroxidation, was increased in patients with diabetes mellitus. ${ }^{28}$ Thus, monitoring changes in either plasma or urinary 8-isoprostane levels can provide an important biochemical link between impaired glycemic control and persistent systemic oxidative stress.

Oxidative (free radical) stress has been implicated as a major underlying mechanism behind many acute and chronic diseases, including type 2 diabetes. ${ }^{29}$ However, the measurement of the levels of free radicals or their end products is complicated. Isoprostanes, which are derived from 
the nonenzymatic peroxidation of arachidonic acid, are now considered to be reliable biomarkers of oxidant stress in the human body. Free 8-isoprostane has been the most commonly measured F2-isoprostane to date and has been used as the gold standard index of in vivo oxidative stress. ${ }^{30}$ Our basic research laboratory routinely measures the free levels of plasma 8-isoprostane as a representative index of oxidative stress in rats experiencing hypomagnesemia or cancer drug toxicity. ${ }^{31-33}$ In patients with type 2 diabetes, elevated levels of 8-isoprostane in urine and blood have been observed. ${ }^{29}$ However, due to patient variability, in a typical clinical study, not all patients with type 2 diabetes display elevated blood isoprostane levels. ${ }^{34}$ In our study, approximately half of the patients had normal plasma 8-isoprostane levels at $\leq 50 \mathrm{pg} / \mathrm{mL}$; on average, the plasma free 8-isoprostane levels in healthy individuals were reviewed and estimated to be $45 \pm 18 \mathrm{pg} / \mathrm{mL} .{ }^{30}$ In our study, approximately one third of our patient cohort $(n=15)$ displayed elevated isoprostane levels at $\geq 80 \mathrm{pg} / \mathrm{mL}$ at the beginning of study. Therefore, we chose this subset of patients to determine whether $\mathrm{Mg}$ supplementation would have an effect. As represented in Figure 2, the average isoprostane level of these 15 patients at the baseline was $91.7 \pm 21.8 \mathrm{pg} / \mathrm{mL}$. At the end of the study, the average 8-isoprostane level was significantly reduced to $71.7 \pm 27.5 \mathrm{pg} / \mathrm{mL}$ ( $p=0.0323$ ), suggesting that for individuals with high indices of oxidative stress, attenuation of the stress was achieved after 3 months. In a separate analysis to compare the treatment effects on changes in isoprostane levels between the obese and nonobese groups ( 25 patients were selected), significant reduction in the isoprostane level was observed at 3 months in the obese group, although nonsignificant decreases were also found in the nonobese group.

\section{Conclusion}

Our data showed that oral Mg-l-lactate replacement therapy after 3 months of treatment with an average dose of 336 mg daily corrected hypomagnesemia and had an antiinflammatory effect in patients with type 2 diabetes. Oral $\mathrm{Mg}$ supplementation significantly increased the intracellular and serum levels. Statistically, clinical improvement in HbAlc and CRP levels was not observed at the end of the study, but a significant decrease in the inflammatory biomarker TNF- $\alpha$ as well as in the oxidative biomarker isoprostane was evident.

We were able to employ a feasible and precise method for assessing intracellular Mg level in noninvasively obtained tissue samples, to routinely determine intracellular $\mathrm{Mg}$ levels. The findings of this clinical trial are important for understanding the role of hypomagnesemia in diabetes patients and may provide support for this therapeutic approach for improving insulin sensitivity in patients with type 2 diabetes. We suggest that $\mathrm{Mg}$ serum levels and, potentially, cellular levels should always be routinely determined for patients with type 2 diabetes and that hypomagnesemia, if present, should be corrected with Mg supplementation. Especially in a country as Kuwait and the Gulf Cooperation Council in general where the major source of drinking water is desalinated water in which Mg may be less available than from regular groundwater presumably leading to a diet low in $\mathrm{Mg} .{ }^{35,36}$

A longer intervention period of at least 4 months and/or higher doses of $\mathrm{Mg}$, or dosage adjustment according to body weight, might be necessary to provide significant changes in HbA1c levels. We hypothesize that a larger clinical trial that includes nonobese and obese participants with type 2 diabetes taking into consideration the average $\mathrm{Mg}$ content in the diet would be feasible. We further recommend studying the extent to which replenishing $\mathrm{Mg}$ stores, particularly intracellular $\mathrm{Mg}$ leads to decreased insulin resistance.

\section{Data availability}

Data related to this clinical trial, de-identified, can be shared with interested researchers immediately after publication including a detailed clinical trial report and the study protocol. All of the individual participant data collected during the trial are stored electronically and can be made available with no end date through contacting the principal investigators of this trial (https://clinicaltrials.gov/ct2/show/NCT01980459).

\section{Acknowledgments}

This study was funded by a grant through the Kuwait Foundation for the Advancement of Science "Magnesium Treatment of Inflammation in Disorders of Glucose Homeostasis (2013-1302-01) and the DDI (RA 2012-013). The study was registered in ClinicalTrials.gov (https://clinicaltrials.gov/ct2/ show/NCT01980459).

The initial encouragement for this clinical trial was provided by late Dr Mahmoud Abdulrahim of the Kuwait Foundation for Advancement of the Sciences whose efforts in facilitating this trial are highly appreciated. The authors express their sincere appreciation to the late Dr Narayanan Nampoory, whose contribution to this work, insight, expertise, and support was of great significance. Dr Nampoory was a coprincipal investigator at study initiation and directed all patient-related aspects ensuring patient safety throughout the trial period. The authors acknowledge the efforts of Dr Osama Alsmadi for initiating and fostering the collaboration with Dr Weglicki at the beginning of the study and for obtaining initial ethical approval for conducting the study at DDI. The authors extend their thanks and appreciation to 
Dr Talal Muzaffar who took over the last clinics of the final visit patients and supported the study team in the screening phase. The authors thank Dr Bassam Bulbanat for conducting the ECGs ensuring patient safety. Our thanks are also extended to Mrs Asmaa Alhubail and the clinical laboratory team at DDI for their blood sample analysis and excellent technical support. The Nursing Department at the DDI is thanked for their assistance in buccal swab collection, and the Tissue Bank Department at DDI is thanked for plasma sample processing and sample storage. The authors thank pharmacists: Amina Alqassar for dispensing the Mg tablets, Naeema Qabandi for assistance in screening, and Mona Awadh for medication review. Ms Hidaya Abdalla is thanked for her assistance in patient recruitment for this study. Dr Adel Ahmed is sincerely thanked for supporting the study team and facilitating this project in the clinical research unit at DDI. Niche Pharmaceuticals Inc. is thanked for the generous donation of Mag-Tab SR (sustained release) tablets for this clinical trial. The authors thank all the patients whose participation made this study possible. Part of this study was presented in an oral presentation and poster at the 2nd World Congress for Clinical Trials in Diabetes (November 2017; Berlin, Germany). The authors would like to thank Enago (www.enago.com) for the English language review.

\section{Author contributions}

NZ coordinated with the study site in Kuwait, led the clinical experiments, and aided in designing the clinical study; obtained, analyzed, and interpreted the data; and wrote, edited, and reviewed the manuscript. NA obtained data, assisted in the clinics, and edited and reviewed the manuscript. ITM contributed to oversight of research parameters/methodology of assays of inflammation and oxidative stress, contributed to writing, interpreted data, and edited and reviewed the manuscript. WBW designed the study; contributed to oversight of patient recruitment, sampling, cellular data collection, and analysis; interpreted the clinical data; and wrote, edited, and reviewed the manuscript. BS conducted EXATEST cellular mineral testing; contributed to the interpretation and oversight of cellular mineral assays; wrote the methodology for the assay; and edited and reviewed the manuscript. All the authors gave final approval of the version to be published. NZ and WW are the guarantors of this work and take responsibility for the contents of this article. All authors contributed toward data analysis, drafting and revising the paper and agree to be accountable for all aspects of the work.

\section{Disclosure}

The authors report no conflicts of interest in this work.

\section{References}

1. Barbagallo M, Di Bella G, Brucato V, et al. Serum ionized magnesium in diabetic older persons. Metabolism. 2014;63(4):502-509.

2. Rodriguez-Moran M, Simental Mendia LE, Zambrano Galvan G, Guerrero-Romero F. The role of magnesium in type 2 diabetes: a brief based-clinical review. Magnes Res. 2011;24(4):156-162.

3. Kao WH, Folsom AR, Nieto FJ, Mo JP, Watson RL, Brancati FL. Serum and dietary magnesium and the risk for type 2 diabetes mellitus: the Atherosclerosis Risk in Communities Study. Arch Intern Med. 1999;159(18):2151-2159.

4. Rude RK. Magnesium deficiency and diabetes mellitus. Causes and effects. Postgrad Med. 1992;92(5):217-219, 222-224.

5. Silver BB. Development of cellular magnesium nano-analysis in treatment of clinical magnesium deficiency. $J$ Am Coll Nutr. 2004;23(6):732S-737S

6. Haigney MC, Silver B, Tanglao E, et al. Noninvasive measurement of tissue magnesium and correlation with cardiac levels. Circulation. 1995;92(8):2190-2197.

7. Simental-Mendia LE, Rodriguez-Moran M, Guerrero-Romero F. Oral magnesium supplementation decreases $\mathrm{C}$-reactive protein levels in subjects with prediabetes and hypomagnesemia: a clinical randomized double-blind placebo-controlled trial. Arch Med Res. 2014;45(4):325-330.

8. Seidell JC, Flegal KM. Assessing obesity: classification and epidemiology. Br Med Bull. 1997;53(2):238-252.

9. Guerrero-Romero F, Rodriguez-Moran M. Magnesium improves the beta-cell function to compensate variation of insulin sensitivity: doubleblind, randomized clinical trial. Eur J Clin Invest. 2011;41(4):405-410.

10. Tosiello L. Hypomagnesemia and diabetes mellitus. A review of clinical implications. Arch Intern Med. 1996;156(11):1143-1148.

11. Rodriguez-Moran M, Guerrero-Romero F. Oral magnesium supplementation improves insulin sensitivity and metabolic control in type 2 diabetic subjects: a randomized double-blind controlled trial. Diabetes Care. 2003;26(4):1147-1152.

12. Veronese N, Watutantrige-Fernando S, Luchini C, et al. Effect of magnesium supplementation on glucose metabolism in people with or at risk of diabetes: a systematic review and meta-analysis of double-blind randomized controlled trials. Eur J Clin Nutr. 2016;70(12):1463.

13. Nadler JL, Malayan S, Luong H, Shaw S, Natarajan RD, Rude RK. Intracellular free magnesium deficiency plays a key role in increased platelet reactivity in type II diabetes mellitus. Diabetes Care. 1992;15(7):835-841.

14. Nadler JL, Buchanan T, Natarajan R, Antonipillai I, Bergman R, Rude R. Magnesium deficiency produces insulin resistance and increased thromboxane synthesis. Hypertension. 1993;21(6 Pt 2):1024-1029.

15. Gommers LM, Hoenderop JG, Bindels RJ, de Baaij JH. Hypomagnesemia in Type 2 Diabetes: A Vicious Circle? Diabetes. 2016;65(1):3-13.

16. Song Y, He K, Levitan EB, Manson JE, Liu S. Effects of oral magnesium supplementation on glycaemic control in Type 2 diabetes: a meta-analysis of randomized double-blind controlled trials. Diabet Med. 2006;23(10):1050-1056.

17. Elin RJ. Status of the determination of magnesium in mononuclear blood cells in humans. Magnesium. 1988;7(5-6):300-305.

18. Huang CL, Kuo E. Mechanism of hypokalemia in magnesium deficiency. J Am Soc Nephrol. 2007;18(10):2649-2652.

19. Whang R, Welt LG. Observations in experimental magnesium depletion. J Clin Invest. 1963;42:305-313.

20. Guerrero-Romero F, Simental-Mendia LE, Hernandez-Ronquillo G, Rodriguez-Morán M. Oral magnesium supplementation improves glycaemic status in subjects with prediabetes and hypomagnesaemia: a double-blind placebo-controlled randomized trial. Diabetes Metab. 2015;41(3):202-207.

21. Guerrero-Romero F, Bermudez-Pena C, Rodriguez-Moran M. Severe hypomagnesemia and low-grade inflammation in metabolic syndrome. Magnes Res. 2011;24(2):45-53. 
22. Pearson TA, Mensah GA, Alexander RW, et al. Markers of inflammation and cardiovascular disease: application to clinical and public health practice: a statement for healthcare professionals from the Centers for Disease Control and Prevention and the American Heart Association. Circulation. 2003;107(3):499-511.

23. Ridker PM. Clinical application of C-reactive protein for cardiovascular disease detection and prevention. Circulation. 2003;107(3):363-369.

24. Sukegawa H, Maekawa Y, Yuasa S, et al. Intensive statin therapy stabilizes C-reactive protein, but not chemokine in stable coronary artery disease treated with an everolimus-eluting stent. Coron Artery Dis. 2016;27(5):405-411.

25. Mora S, Ridker PM. Justification for the Use of Statins in Primary Prevention: an Intervention Trial Evaluating Rosuvastatin (JUPITER) - can C-reactive protein be used to target statin therapy in primary prevention? Am J Cardiol. 2006;97(2A):33A-41A.

26. Haendeler J, Hoffmann J, Zeiher AM, Dimmeler S. Antioxidant effects of statins via S-nitrosylation and activation of thioredoxin in endothelial cells: a novel vasculoprotective function of statins. Circulation. 2004;110(7):856-861.

27. Davignon J, Jacob RF, Mason RP. The antioxidant effects of statins. Coron Artery Dis. 2004;15(5):251-258.

28. Davi G, Ciabattoni G, Consoli A, et al. In vivo formation of 8-isoprostaglandin f2alpha and platelet activation in diabetes mellitus: effects of improved metabolic control and vitamin E supplementation. Circulation. 1999;99(2):224-229.
29. Kaviarasan S, Muniandy S, Qvist R, Ismail IS. F(2)-isoprostanes as novel biomarkers for type 2 diabetes: a review. J Clin Biochem Nutr. 2009;45(1):1-8.

30. van 't Erve TJ, Kadiiska MB, London SJ, Mason RP. Classifying oxidative stress by F2-isoprostane levels across human diseases: a metaanalysis. Redox Biol. 2017;12:582-599.

31. Mak IT, Chmielinska JJ, Kramer JH, et al. Loss of neutral endopeptidase activity contributes to neutrophil activation and cardiac dysfunction during chronic hypomagnesemia: protection by substance P receptor blockade. Exp Clin Cardiol. 2011;16(4):121-124.

32. Mak IT, Kramer JH, Chmielinska JJ, Spurney CF, Weglicki WB. EGFRTKI, erlotinib, causes hypomagnesemia, oxidative stress, and cardiac dysfunction: attenuation by $\mathrm{NK}-1$ receptor blockade. J Cardiovasc Pharmacol. 2015;65(1):54-61.

33. Mak IT, Kramer JH, Chen X, Chmielinska JJ, Spurney CF, Weglicki WB. Mg supplementation attenuates ritonavir-induced hyperlipidemia, oxidative stress, and cardiac dysfunction in rats. Am J Physiol Regul Integr Comp Physiol. 2013;305(10):R1102-R1111.

34. Casoinic F, Sampelean D, Buzoianu AD, Hancu N, Baston D. Serum Levels of oxidative stress markers in patients with type 2 diabetes mellitus and non-alcoholic steatohepatitis. Rom J Intern Med. 2016;54(4):228-236.

35. Fadlelmawla A, Al-Otaibi M. Analysis of the water resources status in Kuwait. J Water Resour Manage. 2005;19(5):555-570.

36. Al-Rashed MF and Sherif MM. Water resources in the GCC countries: an overview. J Water Resour Manage. 2000;14(1):59-75. 


\section{Supplementary materials}

At study initiation, 62 patients were allocated to receive magnesium L-lactate tablets. Eight participants were excluded prior to receiving the intervention, and seven participants dropped out of the study. Reasons for exclusion were as follows: abnormal ECGs (four participants), two participants were no longer interested in participating, one participant left the country, and one participant concurrently started another magnesium supplement (magnesium oxide tablets). Seven participants dropped out of the study and discontinued taking the intervention. Reasons for dropping out were as follows: three participants could not commit to sample collection appointments, two participants travelled abroad during the study period, and two participants were doubtful of the benefit of the tablets.

Table SI Demographics and clinical characteristics of the study population at baseline and at the end of 3-month therapy

\begin{tabular}{|c|c|c|c|c|c|c|}
\hline & \multicolumn{2}{|c|}{ Patients $(n=47)$} & \multicolumn{2}{|c|}{ Obese group $(n=32)$} & \multicolumn{2}{|c|}{ Nonobese group $(n=15)$} \\
\hline & Baseline & End & Baseline & End & Baseline & End \\
\hline Age & $52 \pm 10.2$ & - & $50.5 \pm 10.6$ & - & $55.2 \pm 8.9$ & - \\
\hline \multicolumn{7}{|l|}{ Gender } \\
\hline Men & 25 & 25 & 14 & 14 & 11 & 11 \\
\hline Women & 22 & 22 & 18 & 18 & 4 & 4 \\
\hline BMI $\left(\mathrm{kg} / \mathrm{m}^{2}\right)$ & $32.32 \pm 5.6$ & $32.8 \pm 5.9$ & $35.2 \pm 4.4$ & $35.7 \pm 4.9$ & $26.2 \pm 1.8$ & $26.7 \pm 1.8$ \\
\hline Weight (kg) & $89.9 \pm 17.3$ & $91.4 \pm 18.5 * *$ & $96.3 \pm 17.1$ & $97.7 \pm 18.6^{*}$ & $76.4 \pm 7.5$ & $77.9 \pm 8.1$ \\
\hline Systolic blood pressure (mmHg) & $128.5 \pm 12.8$ & $135 \pm 14.5 * * *$ & $130.1 \pm 13.2$ & $136.9 \pm 14.5^{* *}$ & $|25 \pm| \mid .4$ & $130.8 \pm 13.9$ \\
\hline Diastolic blood pressure $(\mathrm{mmH})$ & $70.5 \pm 12$ & $72 \pm 15.9$ & $70.6 \pm 12.8$ & $71.4 \pm 17.5$ & $70.3 \pm 10.5$ & $73.3 \pm 12.1$ \\
\hline Heart rate & $83.9 \pm 10.5$ & $83.5 \pm 12.8$ & $83.4 \pm I I .3$ & $84.8 \pm I \mid .4$ & $85 \pm 8.7$ & $80.6 \pm 15.4$ \\
\hline Creatinine $(\mu \mathrm{mol} / \mathrm{L})$ & $73.5 \pm 14.6$ & $76.6 \pm 20.2$ & $72 \pm 13.2$ & $76.1 \pm 20.9$ & $76.7 \pm 17.4$ & $77.5 \pm 19$ \\
\hline eGFR $\left(\mathrm{mL} / \mathrm{min} / 1.73 \mathrm{~m}^{2}\right)$ & $88.6 \pm 17.1$ & $85.5 \pm 19.9$ & $89.3 \pm 17.1$ & $84.9 \pm 20.2^{*}$ & $87.2 \pm 17.8$ & $86.7 \pm 19.8$ \\
\hline
\end{tabular}

Notes: Values are mean $\pm S D$. *p-value between baseline and end. Creatinine $(\mathrm{Cr})$ normal level: $71-115$ ( $\mu \mathrm{mol} / \mathrm{L})$.

Abbreviations: BMI, body mass index; eGFR, estimated glomerular filtration rate. 


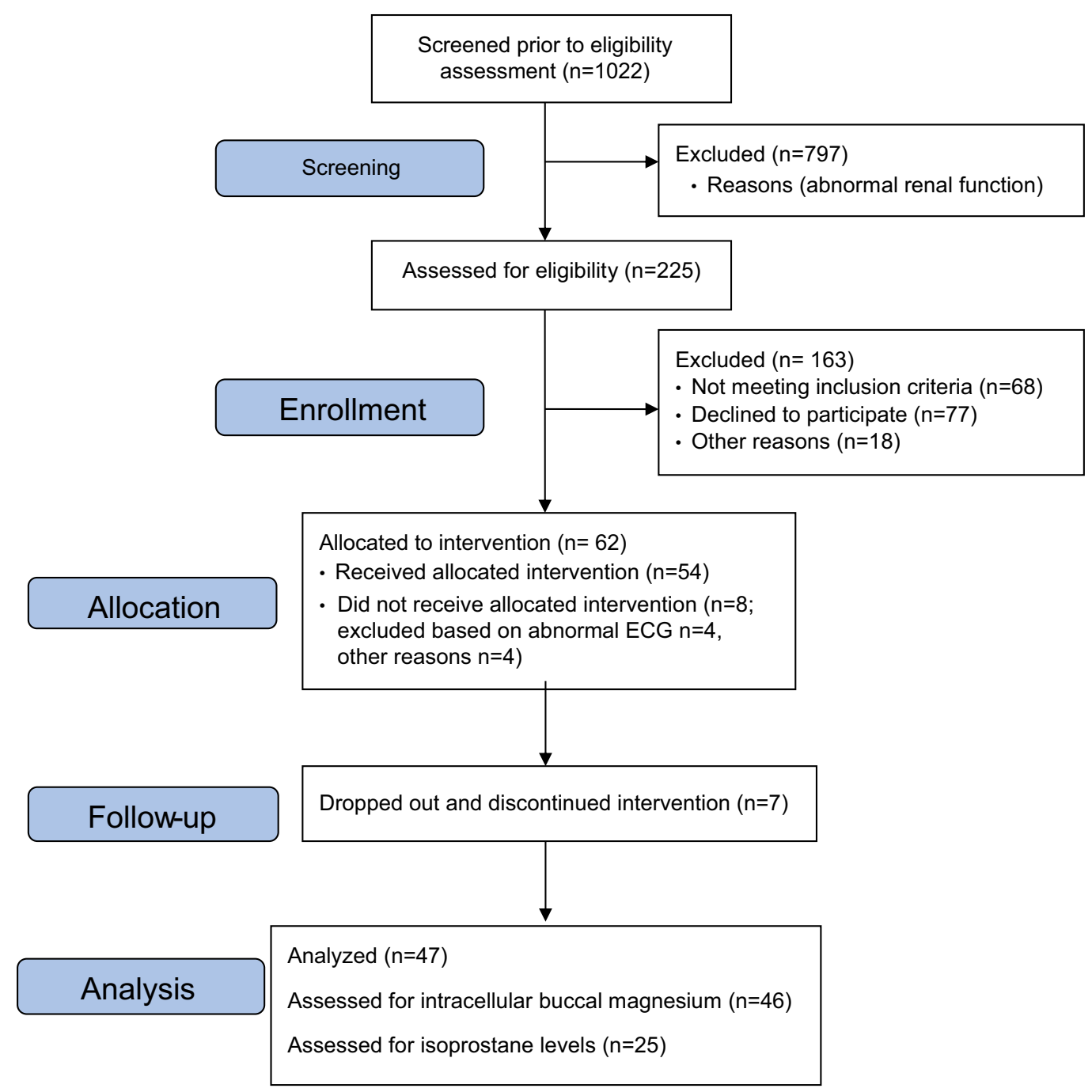

Figure SI The study flow diagram, detailing the phases of the trial including enrollment, intervention allocation, follow-up, and data analysis.

\section{Publish your work in this journal}

Diabetes, Metabolic Syndrome and Obesity: Targets and Therapy is an international, peer-reviewed open-access journal committed to the rapid publication of the latest laboratory and clinical findings in the fields of diabetes, metabolic syndrome and obesity research. Original research, review, case reports, hypothesis formation, expert opinion and commentaries are all considered for publication. The manuscript management system is completely online and includes a very quick and fair peer-review system, which is all easy to use. Visit http://www.dovepress.com/testimonials.php to read real quotes from published authors.

Submit your manuscript here: https://www.dovepress.com/diabetes-metabolic-syndrome-and-obesity-targets-and-therapy-journal 